\title{
A Study of Traditional and Surrounding Gate MOSFET using TCAD Simulations
}

\author{
Neeraj Gupta*, Janak B. Patel and A. K. Raghav \\ ECE, Amity School of Engineering and Technology, Gurgaon, Haryana, India; neerajsingla007@gmail.com, \\ janakbpatel71@gmail.com, akraghava@gmail.com
}

\begin{abstract}
In this paper, Traditional and surrounding gate MOSFET are simulated using TCAD Silvaco and report the effect of multigate transistor. As the channel length of transistors is scaling down, a traditional MOSFET suffers from short channel effects and degrade the device performance. The different parameter such as threshold voltage, area, subthreshold slope, channel length modulation and leakage current are extracted for traditional and surrounding gate MOSFET. The parameters obtained from surrounding gate MOSFET is compared with a traditional MOSFET of the same dimension. The Results show that better performance was obtained for surrounding gate MOSFET. Its current drive capability is more as compared to traditional MOSFET .However, benefits of achieving superior performance with surrounding gate MOSFET reduces in terms of poor RF performance. Hence, this paper concludes that surrounding gate MOSFET have huge potential to be a promising contender to the traditional MOSFETs for making of future generation low-power high speed devices which could minimize the occupied area.
\end{abstract}

Keywords: Channel Length Modulation, Leakage Current, Subthreshold Slope, Surrounding Gate MOSFET, Threshold Voltage,

\section{Introduction}

The today's VLSI fulfils the demand of devices with higher speed, higher packing density, low power dissipation and energy efficient. Several short channel effect such as sub threshold swing, velocity saturation, threshold voltage roll-off (short-channel effect), hot electron effect, increase in leakage current and Drain Induced Barrier Lowering (DIBL) and Gate Induced Drain Lowering (GIDL).So it is important to take the device approaches in other ways to minimize not only the channel length of the device but also the channel width for the planar transistor to achieve higher packing density. Surrounding Gate Transistor (SGT) is a non planer device in which the channel is in the vertical direction. It is a device, whose gate electrode surrounds the silicon pillar. It reduces the area for the transistors. It increases packing density up to $50 \%$ to the surrounding gate $\mathrm{MOSFET}^{\mathrm{l}-3}$.
This paper is partitioned into five parts: Part-I covered the introduction of SGT, Part-II introduce devices structures of SGMOSFET and Traditional MOSFET, The analysis of electrical characteristics with the help of output drain and transfer characteristics of SGMOSFET and traditional MOSFET are described in Part-III. Part-IV includes the results and Part- $\mathrm{V}$ covered the conclusion and future work.

\section{Device Structure}

Surrounding Gate (SG) MOSFET is a device in which gate is surrounded from all the sides so increases the current driving capability. Fig 1 \& Fig 2 shows the device structure of the SG MOSFET and the traditional MOSFET.

The source and drain doping concentration is about $1 \mathrm{X} 10^{18} \mathrm{~cm}^{-3}$ and channel doping concentration is about $1 \mathrm{X} 10^{16} \mathrm{~cm}^{-3}$, channel length is $240 \mathrm{~nm}$ and with a oxide thickness of $20 \mathrm{~nm}$ for SG MOSFET and the traditional MOSFET.

${ }^{*}$ Author for correspondence 


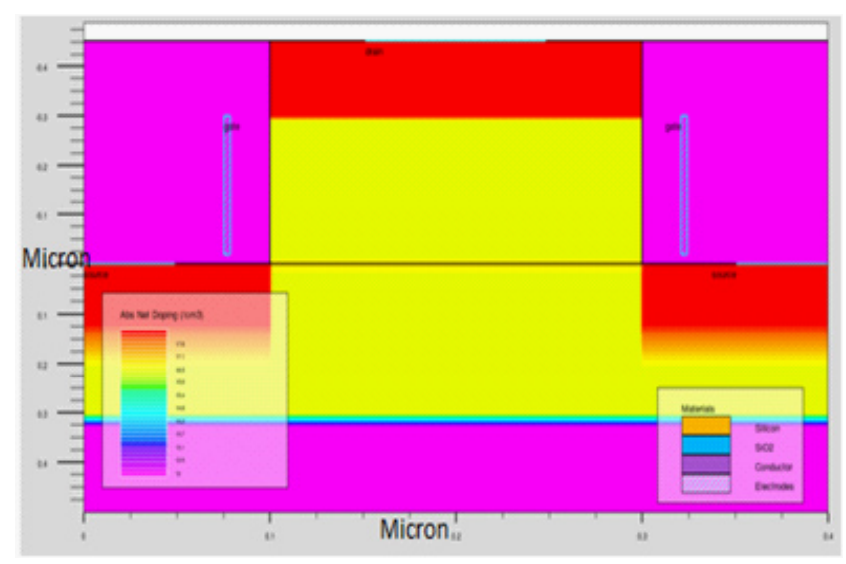

Figure 1. Device Structure of Surrounded Gate MOSFET.

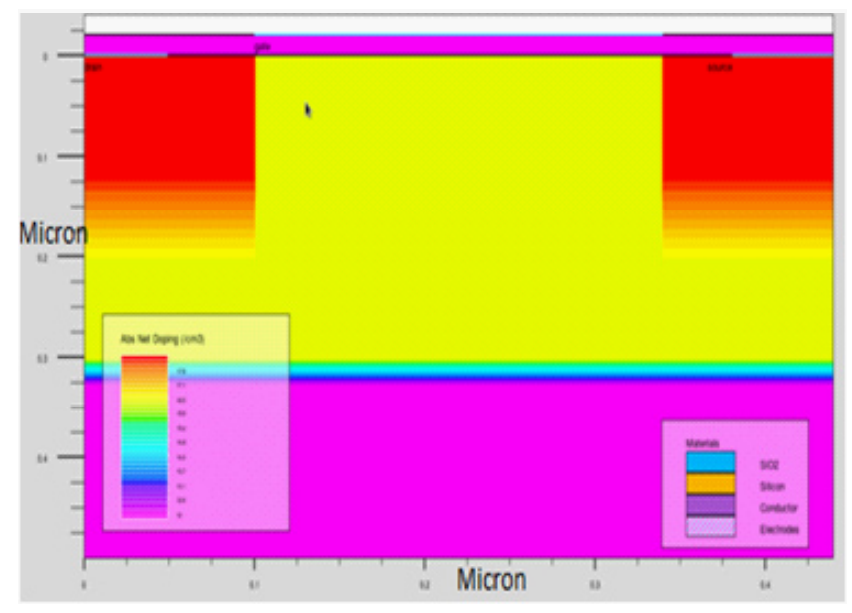

Figure 2. Device Structure of Traditional MOSFET.

\section{Analysis of Electrical Characteristics}

\subsection{Transfer Characteristics}

Fig 3 \& Fig 4 shows the transfer characteristics of the SG MOSFET and the traditional MOSFET.

The SGMOSFET has a larger current driving capacity than the traditional MOSFET as demonstrates from transfer characteristics. This is mainly due to the channel is surrounded by the gate from all sides in SGMOSFET and presence of multigate in SGMOSFET. The SGMOSFET required smaller on voltage instead of the traditional MOSFET. It means that the Gate electrode has a higher control over the conducting channel in a SGMOSFET. The device performance ratio i.e. Ion / Ioff of the SGMOSFET is more as compared to traditional MOSFET $\frac{13,14}{2}$. It means

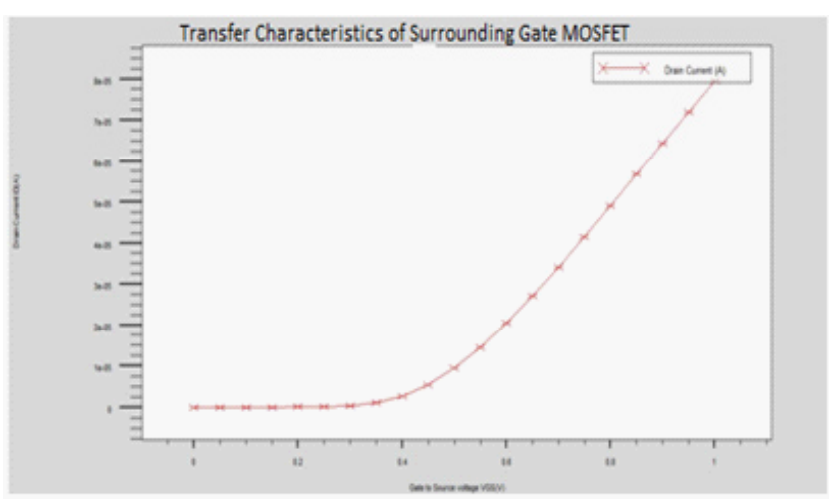

Figure 3. Transfer Characteristics of Surrounded Gate MOSFET.

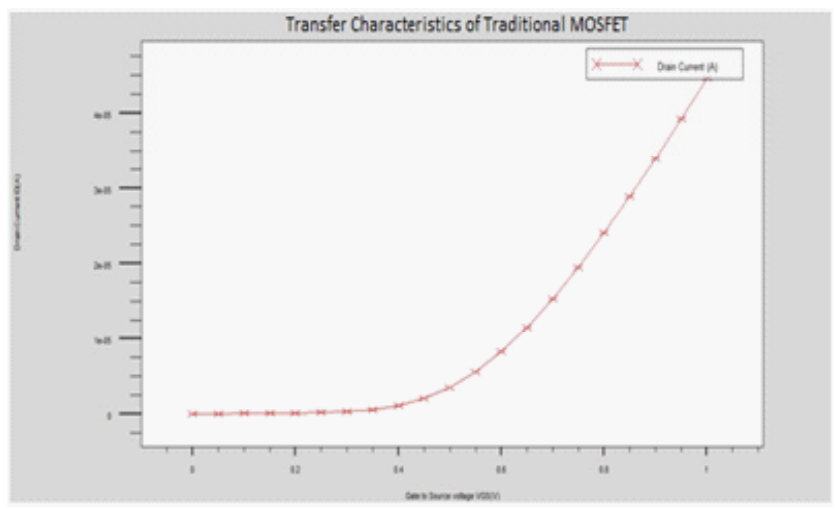

Figure 4. Transfer Characteristics of Traditional MOSFET.

leakage current is also very small in SGMOSFET and it reduces the power dissipation ${ }^{4-7}$. As shown in the Fig 3 \& 4 that SGMOSFET has a smaller sub-threshold swing (SS) than a traditional MOSFET.

\subsection{Output Characteristics}

Fig 5 \& Fig 6 shows the output drain characteristics of the SGMOSFET and for the traditional MOSFET are plotted with the help of TCAD silvaco ${ }^{\circ}$.

The output drain characteristics are plotted between drain current and drain voltage at some constant value of the gate voltage. The output drain characteristics are very much similar in nature as that of a traditional MOSFET. The output drain characteristics revels that, the current driving capacity of SGMOSFET is larger than the traditional MOSFET. The SGMOSFET on resistance (Ron) is small in the linear region instead of a traditional MOSFET 9 -11,14. 


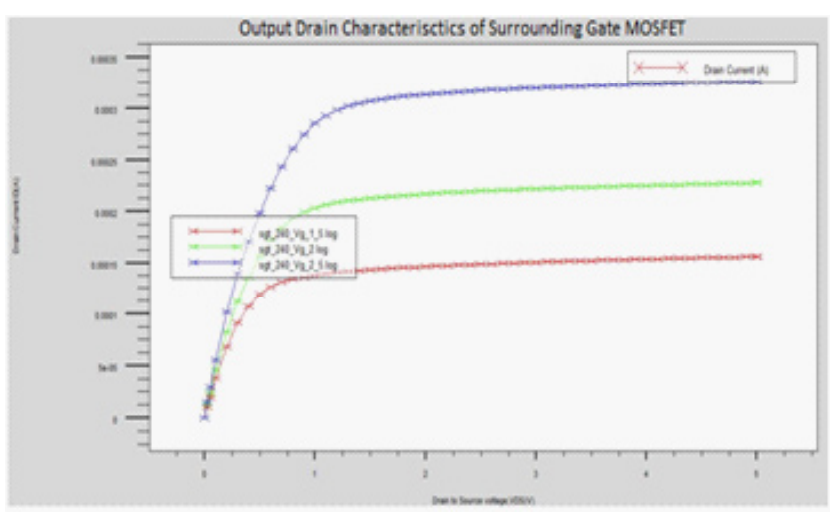

Figure 5. Output Drain Characteristics of SG MOSFET.

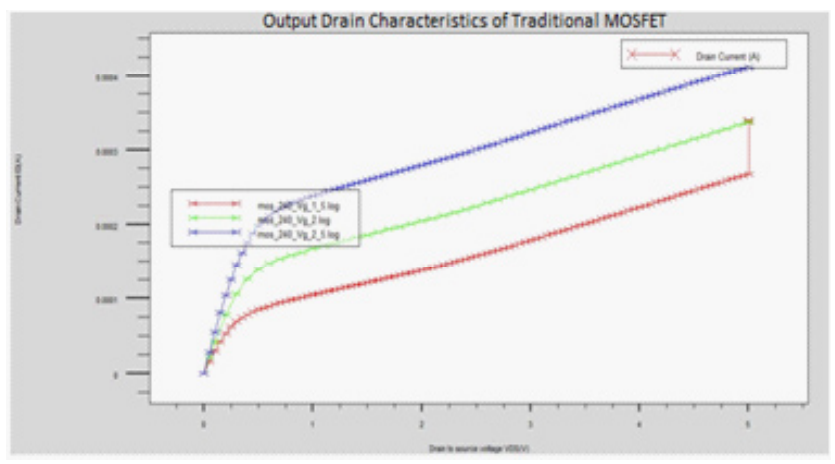

Figure 6. Output Drain Characteristics of Traditional MOSFET.

\section{Results}

1. Area covered by SGMOSFET $=$ width $(\mathrm{W})$, since the channel is in the vertical direction.

Area covered by MOSFET $=\mathrm{L}^{\star} \mathrm{W}=240 \mathrm{~nm}^{\star} \mathrm{W} \mathrm{m}^{2}$

So area on wafer can be saved using SGMOSFET, while having the same current driving capability.

2. Reduced channel length modulation

$$
\mathrm{I}_{\mathrm{DS} 1} / \mathrm{I}_{\mathrm{DS} 2}=\left(1+\lambda \mathrm{V}_{\mathrm{DS} 1}\right) /\left(1+\lambda \mathrm{V}_{\mathrm{DS} 2}\right)
$$

The output drain characteristics of the SGMOSFET is shown by red line and the output drain characteristics of the traditional MOSFET is shown by green line as depicted in fig. 7.

As the channel length modulation (CLM) is diminished in SGT, which make it best for analog circuit design $\underline{16}$.

The Transfer characteristics of the SGMOSFET are shown by red line and the Transfer characteristics of the traditional MOSFET is shown by green line as depicted in Fig. 8.

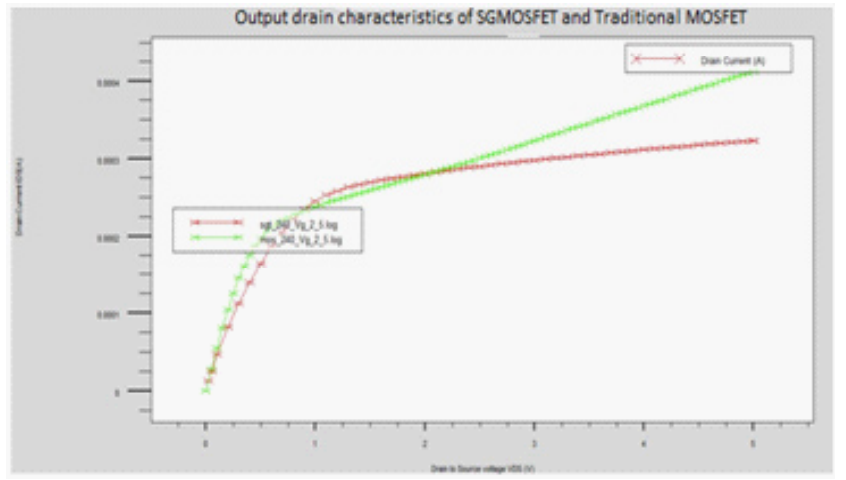

Figure 7. Output drain characteristics of SGMOSFET and Traditional MOSFET for $\mathrm{V}_{\mathrm{GS}}=2.5 \mathrm{~V}$.

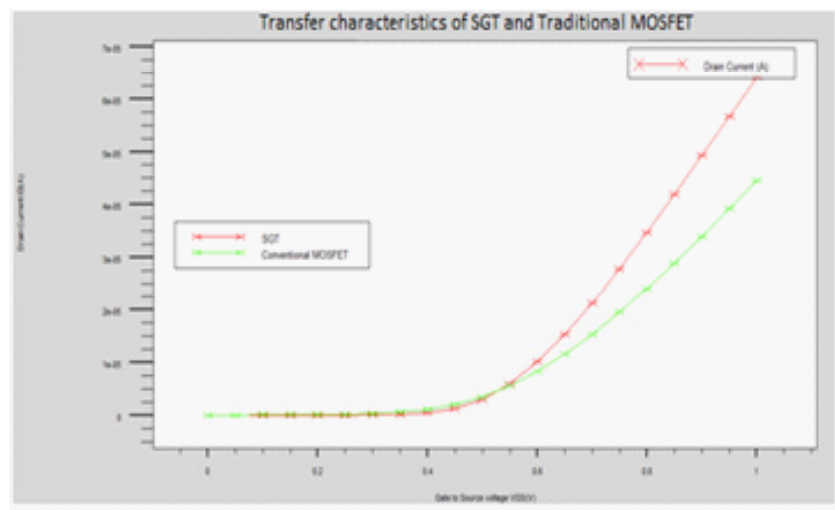

Figure 8. Transfer characteristics of SGT and Traditional MOSFET for $\mathrm{V}_{\mathrm{DS}}=1 \mathrm{~V}$.

Table 1. Parameters to find Channel length modulation

\begin{tabular}{|c|c|c|c|}
\hline Device & $\mathrm{V}_{\mathrm{DS}}(\mathrm{V})$ & $\mathrm{I}_{\mathrm{DS}}(\mathrm{A})$ & $\lambda\left(\mathrm{V}^{-1}\right)$ \\
\hline $\begin{array}{c}\text { Surrounded } \\
\text { Gate MOSFET }\end{array}$ & 3 & 0.000295 & \multirow{2}{*}{0.06} \\
\cline { 2 - 3 } & 4 & 0.00031 & \\
\hline $\begin{array}{c}\text { Traditional } \\
\text { MOSFET }\end{array}$ & 3 & 0.000325 & \multirow{2}{*}{0.28} \\
\cline { 2 - 3 } & 4 & 0.000375 & \\
\hline
\end{tabular}

3. Threshold Voltage

Threshold voltage of Traditional $\operatorname{MOSFET}\left(\mathrm{V}_{\mathrm{T}}\right)=$ $0.45 \mathrm{~V}$

Threshold voltage of SGT $\left(\mathrm{V}_{\mathrm{T}}\right)=0.4 \mathrm{~V}$

The SGMOSFET having a smaller $\mathrm{V}_{\mathrm{T}}$ than the traditional MOSFET as given above. It means that the 
Gate electrode has a more control over the channel in a SGMOSFET 15,17 .

4.Improved Sub-threshold slope and reduced leakage

Fig. 9 shows the plot between $\log \left(I_{D}\right) V_{s} V_{G S}$ for SGMOSFET and Traditional MOSFET for $\mathrm{V}_{\mathrm{DS}}=1 \mathrm{~V}$.

- Sub threshold slope $(\mathrm{SS})=\frac{\mathbf{d V}_{\mathrm{GS}}}{\mathbf{d}\left(\log \mathbf{I}_{\mathbf{D}}\right)} \mathrm{mV} /$ decade

It is voltage required to change in one decade of current, so SS is less required for fast switching operation $\frac{14,15}{}$.

$\mathrm{SS}$ of $\mathrm{SGT}=90 \mathrm{mV} /$ decade

SS of planer MOSFET $=160 \mathrm{mV} /$ decade

Therefore it can be concluded that SGT is faster than traditional MOSFET.

- Leakage current ${ }^{17}$

From the plot it can be seen that $\mathrm{I}_{\mathrm{D}}$ when $\mathrm{V}_{\mathrm{GS}}=0 \mathrm{~V}$ at $\mathrm{V}_{\mathrm{DS}}=1 \mathrm{~V}$ is the order of $10^{-12}$ for SGT and $10^{-8}$ for MOSFET.

So SGMOSFET required smaller area, low threshold voltage, reduced channel length modulation, Leakage current and Improved Sub-threshold slope ${ }^{12}$.

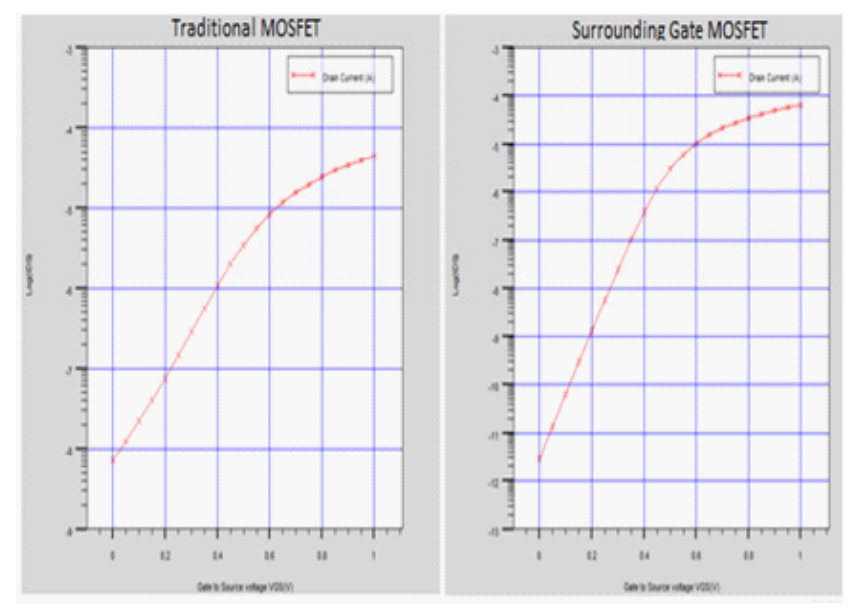

Figure 9. $\log \left(\mathrm{I}_{\mathrm{D}}\right)$ Vs $\mathrm{V}_{\mathrm{GS}}$ plot of SGMOSFET and Traditional MOSFET for $\mathrm{V}_{\mathrm{DS}}=1 \mathrm{~V}$ (a)MOSFET (b) SGMOSFET.

Table 2. Parameters to find Sub-threshold slope

\begin{tabular}{|c|c|c|c|}
\hline Device & $\mathrm{V}_{\mathrm{GS}}(\mathrm{V})$ & $\log \left(\mathrm{I}_{\mathrm{DS}}\right)$ & $\begin{array}{c}\text { Sub-threshold } \\
\text { slope }\end{array}$ \\
\hline $\begin{array}{c}\text { Surrounded } \\
\text { Gate MOSFET }\end{array}$ & 0.26 & 8 & \multirow{2}{*}{$90 \mathrm{mV} /$ decade } \\
\cline { 2 - 3 } & 0.35 & 7 & \\
\hline $\begin{array}{c}\text { Traditional } \\
\text { MOSFET }\end{array}$ & 0.225 & 7 & \multirow{2}{*}{$160 \mathrm{mV} /$ decade } \\
\cline { 2 - 3 } & 0.385 & 6 & \\
\hline
\end{tabular}

\section{Conclusion and Future Work}

SGMOSFET has been adopted for making future devices, which could minimize the occupied area and also reduce the power dissipation. As the channel is surrounded by the gate so we get better electrical control over the MOSFET which is visible through the small sub-threshold slope compare to traditional MOSFETs. There is only manufacturing complexity which increases the cost of fabrication leads another technology like FinFET which fabrication is more similar to planer process.

\section{References}

1. Takato H, Sunouchi K, Okabe N, Nitayama A, Hieda K, Horiguchi F, Masuoka F. Impact of Surrounding Gate Transistor (SGT) for Ultra-High-Density LSI's. IEEE Transactions on Electron Devices. 1991 Mar; 38(3).

2. Subrahmanyam B, Jagadesh Kumar M. Department of Electrical Engineering, Indian Institute of Technology. Recessed source concept in nanoscale vertical surrounding gate (VSG) MOSFETs for controlling short-channel effects, New Delhi 110016 India, Physica E. 2009; 41:671-6

3. Gupta N, Raghav AK, Kushwaha AK. A study on multi material gate all around SOI MOSFET. International Journal of Technological Exploration and Learning. 2014 Jun; 3(3).

4. Solankia T, Parmar N. A Review paper: A Comprehensive study of junctionless transistor. National Conference on Recent Trends in Engineering and Technology, B.V.M. Engineering College, V.V. Nagar, Gujarat, India. 2011 May13-14.

5. Chen C-Y, Lin J-T, Chiang M-H. Comparative Study of Process Variations in Junctionless and Traditional DoubleGate MOSFETs, IEEE. 2013.

6. Lakshmi B, Srinivasan R. Investigation of $\mathrm{ft}$ and nonquasi-static delay in traditional and junctionless multigate transistors using TCAD simulation. ARPN Journal of Engineering and Applied Sciences. 2012 Jul; 7(7).

7. Kim SY et al. Design and Analysis of Sub-10 nm Junctionless Fin-Shaped Field-Effect Transistors. Journal of Semiconductor Technology and Science. 2014 Oct; 14(5).

8. SILVACO International, ATLAS User 's Manual, 2012 Apr.

9. Gupta SK, Pathak GG, Das D, Sharma C. Double Gate MOSFET and its Application for Efficient Digital Circuits. International Conference on Electronics Computer Technology, IEEE. 2011. Doi: 10.1109/ ICECTECH.2011.5941650.

10. Gupta N, Patel JKB, Raghav AK. A study on roadmap for future multi gate SOI mosfet. International Journal of Engineering Research. 2015 Jan; 3(1):1-7. 
11. The International Technology Roadmap for Semiconductors, 2007.

12. Gupta N, Patel JKB, Raghav AK. A Study of Conventional and Junctionless MOSFET Using TCAD Simulations. International Conference on Advanced Computing and Communication Technologies, IEEE. 2015. Doi: 10.1109/ ACCT.2015.51.

13. Fashtami TN, Seyed Ali SZ. Performance Investigation of Gate-All-Around Nanowire FETs for Logic Applications. Indian Journal of Science and Technology. 2015 Feb; 8(3):231-6.

14. Verma JHK, Haldar S, Gupta RS, Gupta M. Modelling and simulation of subthreshold behaviour of cylindrical surrounding double gate MOSFET for enhanced electrostatic integrity. Super lattices and Microstructures. 2015 Sep; 354-64.
15. Yu F, Deng W, Huang J, Ma X, Chen S. An Explicit PhysicsBased I -V Model for Surrounding-Gate Polysilicon Transistors. IEEE Transactions on Electron Devices. 2016 Mar; 63(3):1059-65.

16. Charles Pravin J, Nirmal D, Prajoon P, Ajayan J. Implementation of nanoscale circuits using dual metal gate engineered nanowire MOSFET with high-k dielectrics for low power applications. Physica E. 2016 Apr; 83:95-100.

17. Xiao Y, Zhang B, Lou H, Zhang L, Lin X. A Compact Model of Subthreshold Current with Source/Drain Depletion effect for the Short-Channel Junctionless Cylindrical Surrounding-Gate MOSFETs. IEEE Transactions on Electron Devices. 2016 May; 63(5):2176-81. 